\title{
Graphs of morphisms of graphs
}

\author{
R. Brown, I. Morris, J. Shrimpton and C.D. Wensley \\ School of Computer Science, Bangor University \\ Dean Street, Bangor, Gwynedd, LL57 1UT, U.K. \\ r.brown@bangor.ac.uk, c.d.wensley@bangor.ac.uk
}

Submitted: Jul 13, 2006; Accepted: Mar 29, 2008; Published: Apr 3, 2008

Mathematics Subject Classification: 05-01, 05C25, $18 \mathrm{D} 35$.

Keywords: graph, digraph, cartesian closed category, topos, endomorphism monoid, symmetry object.

\begin{abstract}
This is an account for the combinatorially minded reader of various categories of directed and undirected graphs, and their analogies with the category of sets. As an application, the endomorphisms of a graph are in this context not only composable, giving a monoid structure, but also have a notion of adjacency, so that the set of endomorphisms is both a monoid and a graph. We extend Shrimpton's (unpublished) investigations on the morphism digraphs of reflexive digraphs to the undirected case by using an equivalence between a category of reflexive, undirected graphs and the category of reflexive, directed graphs with reversal. In so doing, we emphasise a picture of the elements of an undirected graph, as involving two types of edges with a single vertex, namely 'bands' and 'loops'. Such edges are distinguished by the behaviour of morphisms with respect to these elements.
\end{abstract}

\section{Introduction}

One aim of this article is to present to the combinatorially minded reader a case for using categorical methods in the theory of graphs. We argue that such methods have advantages in leading to new approaches, new methods and wider analogies, and so in giving a guide to handling more complex situations, such as labelled graphs. The spirit of this method is to study constructions on graphs in terms of the relation to all graphs in the given category, via the agreed morphisms of graphs.

The basic analogy is between the theory of sets and the theory of graphs. A difficulty for such an analogy is that the theory of sets is usually based on the notion of membership, 
which bifurcates for graphs since a graph has not only vertices, but also between vertices it has edges (in the undirected case) or arcs (in the directed case). What then should be a 'member' of a graph?

To make such an analogy, we recognise that analogies in mathematics are generally not between objects themselves but between the relations between objects, and these relations are dealt with by abstraction of structure and axiomatisation of this structure. The use of category theory for this theme is discussed in [6].

Relations between sets are well described by the notion of function, and so it is sensible to discuss the properties of the category, Set, of sets and functions between sets. These properties were developed by Lawvere and Tierney as those of an elementary topos, and publicised initially in, for example, [9, 28]. In particular, Set has a subobject classifier (given by the usual characteristic function of a subset); has finite limits (which includes the notion of product $A \times B$ ); and is cartesian closed so that, analogously to the rule of indices $c^{a b}=\left(c^{b}\right)^{a}$, there is an exponential bijection

$$
\operatorname{Set}(A \times B, C) \cong \operatorname{Set}(A, \operatorname{Set}(B, C)) \text {. }
$$

(exp-sets)

Here a function $f: A \times B \rightarrow C$ has an 'adjoint' $\hat{f}: A \rightarrow \operatorname{Set}(B, C)$ where $\hat{f}(a)$ is the function which maps $b$ to $f(a, b)$, so that a function of two variables is regarded as a variable function of one variable.

By analogy, for a category Dgph of directed graphs, in which $\operatorname{Dgph}(B, C)$ denotes the set of morphisms $B \rightarrow C$ of directed graphs, we need also a directed graph $\operatorname{DGPH}(B, C)$ with $\operatorname{Dgph}(B, C)$ as its set of vertices, and an exponential law

$$
\operatorname{Dgph}(A \times B, C) \cong \operatorname{Dgph}(A, \operatorname{DGPH}(B, C))
$$

(exp-graphs)

Note that for sets there is no distinction between Set and SET since abstract sets have no structure.

It turns out that we can define several categories of undirected graphs and of directed graphs so that each forms an elementary topos: thus these categories have properties analogous to properties of the category Set, and this allows for intuition from Set to suggest constructions and theorems on graphs.

One such construction is the notion of automorphism graph. The automorphisms of a set $X$ form a group $\operatorname{Aut}(X)$, namely another set with a multiplication, identity and inverses satisfying the usual axioms. Analogously, not only should there be a group $\operatorname{Aut}(D)$ of automorphisms of a graph $D$, but also an automorphism graph $\operatorname{AUT}(D)$, so that $\operatorname{AUT}(D)$ is both a group and a graph, with vertex set $\operatorname{Aut}(D)$. The graphical nature of $\operatorname{AUT}(D)$ gives a notion of adjacency between automorphisms, and in particular the notion of inner automorphism, defined as an automorphism of $D$ adjacent to the identity morphism. These ideas were explored by Shrimpton in [21, 22, 23], for the case of directed 
graphs, where he described inner automorphisms in graph-theoretic terms using the notion of inner subgraph. See also [3].

In order to set up categories of graphs or of digraphs we have to define morphisms. It turns out that the notion of morphism of directed graphs has two fairly definitive answers. If we choose to allow morphisms to map an arc to a vertex, then we should work with the category of reflexive graphs, where each vertex has a distinguished, associated loop. This case will be our main concern. If we choose not to allow morphisms to map an arc to a vertex we obtain the category of irreflexive graphs. Both of these categories are toposes (we drop the word elementary), and so it is easy to formulate new notions by direct analogy with the topos Set of sets. We will explain what this means, with examples, in Sections 1 and 3. The distinction between the two toposes is eloquently argued by Lawvere in [15].

We shall also explore, in Sections 5 and 7, corresponding notions in the undirected case. The question of what should be an undirected graph, and morphisms of such graphs, leads to the question of the properties of the corresponding category. It turns out that the most transparent and simple definition does not yield a cartesian closed category. This makes it difficult to decide what should be (if at all) the automorphism object $\operatorname{AUT}(G)$ of such an undirected graph $G$, since we cannot then ensure that $\operatorname{AUT}(G)$ has the structure of both an undirected graph and a group. In particular there is not a clear notion of composition $\operatorname{AUT}(G) \times \operatorname{AUT}(G) \rightarrow \operatorname{AUT}(G)$.

There is however an alternative definition of undirected graphs and their morphisms, namely to consider an undirected graph as a directed graph with a reversal. This is the approach taken by Bumby and Latch in [7] and in several other sources, and does yield a topos Rdgph.

We now point out an unexpected consequence. We have a standard picture of an undirected graph with one edge and two vertices as

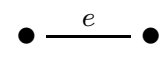

Considered as a directed, reflexive graph with involution, the picture is

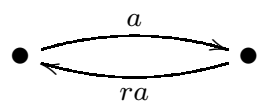

where the reversal $r$ fixes both vertices and interchanges the arcs.

We expect the operation of identifying a pair of vertices to give a morphism of graphs. For (2) this operation gives a directed graph with one vertex and two loops.

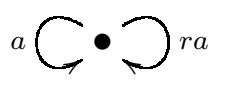

This leads us to consider which of the following pictures should correspond to identifying the two vertices in the graph in (1):

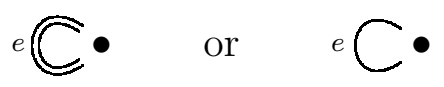

THE EleCtronic Journal of COMBInAtorics 15 (2008), \#A1 
We prefer the first picture, which we call a band, and regard the second picture, which we call a loop, as obtained by a further identification ${ }^{1}$ - it corresponds to a directed loop $a$ with $r a=a$. In the first case, when defining a morphism, an edge in the domain may map to an edge, band or loop in the range; a band may map to a band or loop; but a loop may only map to a loop. This distinction is discussed in more detail in counterexample 5.1 .

In this article, in order to distinguish clearly between the various cases, edges and arcs always have two distinct vertices. Thus our intuitive picture of an undirected graph should (from this viewpoint) consist not only of vertices, edges and loops but also bands, corresponding to the fact that the edges give rise to unordered pairs of vertices. It just happens that most of the graphs studied by graph theorists have no bands and often no loops. Once this extension of the usual notion of undirected graph is made, we may easily define the notion of morphism of an undirected graph as above, and obtain a topos Ugph of reflexive undirected graphs, which is equivalent to the topos Rdgph of reflexive digraphs with reversal.

The principal advantage of Ugph over Rdgph is that the pictures of graphs, and the lists of elements, are simpler. The advantage of Rdgph over Ugph is that it is easier to show that Rdgph is a topos. In either of these toposes we have a clear notion of automorphism object $\operatorname{AUT}(G)$, which is a group in this category.

If we decide that pairs of loops in the digraph, as in (3), should be identified to a (single) loop in the associated graph, then we are changing our topos of undirected graphs to a reflexive subcategory Gph of Ugph. If $A$ is an object of this subcategory, then $\operatorname{AUT}(A)$ is still an object of Ugph, but not necessarily an object of Gph. We give an example of this in Section 5. The reflection $\rho: \operatorname{Rdgph} \rightarrow$ Gph does not preserve products, as we show, and so $\rho(\operatorname{AUT}(A))$ does not necessarily inherit a group structure.

Topos theory is a well-developed subject (see, for example, [14]), but even basic facts, such as "presheaves form a topos", can be very illuminating when applying standard topos-theoretic results to specific combinatorial applications.

A further advantage of this topos-theoretic approach is that more complicated situations can also be handled by exploiting known properties of elementary toposes. For example, a labelled graph $G$ can be considered as a morphism of graphs $f: G \rightarrow L$ where the graph $L$ is a collection of loops at a single vertex (but it could be more general). The theory of toposes [28] tells us that the category of morphisms over $L$ is also a topos, and so we have a notion of automorphism object $\operatorname{AUT}_{L}(G)$ for a labelled graph, described explicitly by general constructions of topos theory. We plan to pursue these generalisations elsewhere.

Other authors have developed and advertised categorical methods for the study of graphs. Gersten (1983) [10], Ribenboim (1983) [19], Stallings (1983) [24], are interested

\footnotetext{
${ }^{1}$ In [16] these are called loops and one lane loops respectively.
} 
in algebraic applications, particularly to group theory. Waller (1976) [27] was interested in topological analogies. Brown [3] advertised the approach of Shrimpton [23] to automorphism structures. Bumby and Latch [7] are interested in seeing how category theory concepts apply to a category of graphs. Hell [12] applies categorical concepts to topics such as chromatic numbers, and Hell and Nešetřil [13] give a wider survey. Waller [26], Vigna [25] and Boldi and Vigna [1] give applications to network problems. Zivaljevic [29] applies related groupoid methods (see also [5]) to graph problems. Golubitsky and Stewart [11] show that many generic features of the dynamics of asymmetric networks can be understood from the groupoid viewpoint. In view of the large work on topos theory, as shown in Johnstone [14], we hope that this approach will also prove fruitful to a range of applications in and of graph theory.

\section{Categories of Graphs}

The spirit of categorical methods in graph theory, as in other areas, is to study a given graph, or a given construction on graphs, by means of its relations to all other graphs. The first step in this process is the notion of morphism between graphs, and so of the category of graphs which is to be studied. It is this aspect of graph theory which is often not emphasised. The next step is the study of categorical constructions in the chosen category of graphs.

An advantage of this method is that it allows a study of the relations between similar classes of objects, for example between various types of graphs, by the use of functors between categories, and of natural transformations between functors. Such an analysis of reflexive and irreflexive digraphs was carried out by Lawvere in [15] from the point of view of topos theory.

In a category $\mathrm{C}$, the set of arrows from an object $x$ to an object $y$ is denoted by $\mathrm{C}(x, y)$, and we write $a: x \rightarrow y$ for $a \in \mathrm{C}(x, y)$. The composition of $a: x \rightarrow y$ and $b: y \rightarrow z$ is written (using the algebraic convention) $a b: x \rightarrow z$. We refer the reader to $[2,17,18]$ for the basic ideas of category theory, but we shall explain many of the results we need.

We first list the various categories of graphs which will be considered. A digraph $D$ has a set $V(D)$ of vertices, a set $U(D)$ of elements, and source and target maps $s, t: U(D) \rightarrow$ $V(D)$. We commonly write an element $e$ as $(e: s e \longrightarrow t e)$, and denote by $D(u, v)$ the set of elements with source $u$ and target $v$. An element $\ell$ is a loop if $s \ell=t \ell$. The elements which are not loops are called arcs, so that $U(D)$ is partitioned as $L(D) \cup A(D)$, the sets of loops and arcs in $D$.

The traditional digraph category Tdgph consists of all directed graphs and morphisms 
between them. Morphisms are pairs of maps which commute with the source and target:

$$
\begin{aligned}
\mu=\left(\mu_{V}, \mu_{U}\right) \in \operatorname{Tdgph}\left(D, D^{\prime}\right) \Leftrightarrow & \mu_{V}: V(D) \rightarrow V\left(D^{\prime}\right), \quad \mu_{U}: U(D) \rightarrow U\left(D^{\prime}\right), \\
& \mu_{V} \circ s=s^{\prime} \circ \mu_{U}, \quad \mu_{V} \circ t=t^{\prime} \circ \mu_{U} .
\end{aligned}
$$

In a reflexive digraph we wish to be able to map elements to vertices. There are two equivalent ways to achieve this. One approach is to include the sets of vertices in the set of elements. The source and target maps become endofunctions on $U(D)$, satisfying $s \circ t=t, t \circ s=s$, and the vertex set is $V(D)=\operatorname{Im} s=\operatorname{Im} t \subseteq L(D)$. A morphism $\mu: D \rightarrow D^{\prime}$ of reflexive digraphs is a function $\mu: U(D) \rightarrow U\left(D^{\prime}\right)$ which preserves source and target, and so maps vertices to vertices, loops to loops, and arcs to any type of element. This is the approach we shall adopt, and we denote the category of reflexive digraphs by Dgph.

The alternative approach is to keep the sets $V(D)$ and $U(D)$ separate, but to add a function $\epsilon: V(D) \rightarrow U(D)$ such that $s \circ \epsilon=t \circ \epsilon=\mathrm{id}$, and require all morphisms to preserve $\epsilon$. Then mapping an arc $a \in D$ to a loop $\epsilon^{\prime} x^{\prime} \in D^{\prime}$ can be thought of as mapping $a$ to the vertex $x^{\prime}$.

We now come to the case of undirected graphs.

For $X$ a set, we denote by $S^{2}(X)$ the set of singleton and two-element subsets of $X$. Any endofunction $\phi$ on $X$ determines an endofunction $\phi_{*}$ on $S^{2}(X)$ by applying $\phi$ to each element.

As indicated in the introduction, we wish to allow reflexive graphs to contain bands, so we also allow these in the irreflexive case. An undirected graph $G$ consists of the following:

- a set $V(G)$ of vertices,

- a set $U(G)$ of elements,

- a boundary map $\partial_{G}: U(G) \rightarrow S^{2}(V(G))$,

- a subset $E(G)=\{e \in U(G):|\partial(e)|=2\} \subseteq U(G)$ of edges,

- a partition of the set of elements $\ell$ of $U(G)$ with $|\partial(\ell)|=1$ into disjoint subsets $B(G), L(G)$, called respectively the sets of bands and loops in $G$.

Our initial intuition was that an undirected graph $G$ should contain a set of loops $L(G)$ with an involutive pairing function $r: L(B) \rightarrow L(B)$; that single loops should be loops with $r(\ell)=\ell$; and that the remaining pairs of loops should form bands $(\ell, r(\ell))$. We later came to realise that a band should be a single element of $G$.

A morphism $\mu: G \rightarrow G^{\prime}$ of undirected graphs is a pair $\left(\mu_{V}, \mu_{U}\right)$ such that

$$
\begin{array}{lll}
\mu_{V}: V(G) \rightarrow V\left(G^{\prime}\right), & \mu_{U}: U(G) \rightarrow U\left(G^{\prime}\right), & \left(\mu_{V}\right)_{*} \circ \partial=\partial^{\prime} \circ \mu_{U}, \\
\mu_{U}(B(G)) \subseteq L\left(G^{\prime}\right) \cup B\left(G^{\prime}\right), & \mu_{U}(L(G)) \subseteq L\left(G^{\prime}\right) . &
\end{array}
$$


The total graph category Tgph consists of all undirected graphs and morphisms between them.

In a reflexive graph $G$ we again take a single underlying set $U(G)$ of elements, which may be vertices, loops, bands, or edges, and a boundary map $\partial: U(G) \rightarrow S^{2}(U(G))$ satisfying $\partial_{*} \circ \partial=\partial$. The vertex set is $V(G)=\{u \in U(G) \mid \partial u=\{u\}\}$. So morphisms are endofunctions on $U(G)$ which preserve the boundary and do not map loops to bands. The reflexive cases provide our main objects of study, and we denote the category of reflexive, undirected graphs by Ugph.

As an aside, we recall that a $k$-colouring of the vertices of $G$ with $k$ colours is an assignment of a colour to each vertex in such a way that adjacent vertices are coloured differently. So, in the irreflexive case, a $k$-colouring is a morphism from $G$ to the complete graph $K_{k}$, where the colours are the vertices of $K_{k}$. The chromatic number $\chi(G)$ is the smallest $k$ such that $G$ has a $k$-colouring. In the reflexive case we allow the image of an edge to be a vertex, violating the colouring condition. Thus the results in this article are not immediately relevant to colouring problems.

Two types of subcategory of Dgph and Ugph are of interest. A graph or digraph is relational if there are no multiple edges or arcs, so that adjacency is a relation on the set of vertices. An irreflexive graph or digraph is strict if there are no loops and no multiple edges or arcs. A reflexive graph or digraph is strict if there are no multiple edges or arcs and the only loops are the vertices. The full subcategories of Ugph and Dgph of strict graphs and digraphs are denoted by Sugph and Sdgph respectively.

\section{Some categorical notions}

We reprise some of the basic notions we shall need. For more information see [2] or [17].

Let $\mathrm{C}$ be a category. Objects $i, t$ of $\mathrm{C}$ are called respectively initial, terminal if, for all objects $A$ of $\mathrm{C}$, the sets $\mathrm{C}(i, A), \mathrm{C}(A, t)$ are singletons. It is easy to prove that any two initial objects are isomorphic, as are any two terminal objects. For all our graph categories the empty graph $\emptyset$, with no vertices and no edges or arcs, is an initial object. We denote by $T$ the graph or digraph with just one vertex and no other element. In the reflexive cases $T$ is a terminal object. In the irreflexive cases the terminal object has one vertex and one loop. For any graph $B$ or digraph $D$, the set $\operatorname{Ugph}(T, B) \operatorname{or} \operatorname{Dgph}(T, D)$ is the set of vertices. Notice that, even at this simple level, we are making analogies between different types of graph.

A product of objects $A_{1}, A_{2}$ in $\mathrm{C}$ is an object $P$ with two arrows $p_{1}: P \rightarrow A_{1}, p_{2}: P \rightarrow$ $A_{2}$ with the property that for any object $Q$ of $\mathrm{C}$ and arrows $q_{1}: Q \rightarrow A_{1}, q_{2}: Q \rightarrow A_{2}$, there is a unique arrow $f: Q \rightarrow P$ such that $q_{1}=f p_{1}, q_{2}=f p_{2}$. It follows that the induced functions on the sets of arrows

$$
\left(p_{1 *}, p_{2 *}\right): \mathrm{C}(Q, P) \rightarrow \mathrm{C}\left(Q, A_{1}\right) \times \mathrm{C}\left(Q, A_{2}\right), f \mapsto\left(f p_{1}, f p_{2}\right)
$$


is a bijection, and the arrow $f$ is entirely determined by its components $f p_{1}$ and $f p_{2}$. This definition determines the triple $\left(P, p_{1}, p_{2}\right)$ up to isomorphism, as is shown by what is known as the 'usual universal argument'. For suppose $\left(P^{\prime}, p_{1}^{\prime}, p_{2}^{\prime}\right)$ is another product of $A_{1}, A_{2}$. Then the morphisms $\left(p_{1}^{\prime}, p_{2}^{\prime}\right)$ determine a morphism $f: P^{\prime} \rightarrow P$ such that $p_{1}^{\prime}=f p_{1}, p_{2}^{\prime}=f p_{2}$. Similarly we get a unique morphism $f^{\prime}: P \rightarrow P^{\prime}$ such that $p_{1}=f^{\prime} p_{1}^{\prime}, p_{2}=f^{\prime} p_{2}^{\prime}$. It follows that, for $i=1,2, f f^{\prime} p_{i}^{\prime}=p_{i}^{\prime}$ and $f^{\prime} f p_{i}=p_{i}$.

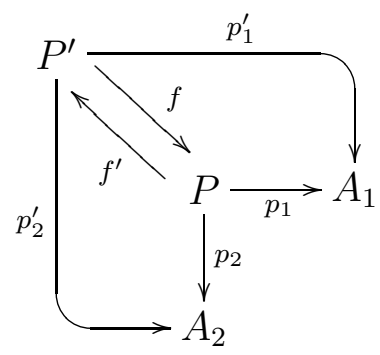

The uniqueness property, with $Q=P, q_{1}=p_{1}, q_{2}=p_{2}$, gives $f^{\prime} f=1_{P}$. Similarly $f f^{\prime}=1_{P^{\prime}}$, so $f, f^{\prime}$ are mutually inverse arrows.

Another notion we will use is the coequaliser. Given two arrows $a, b: A \rightarrow B$ in $\mathrm{C}$, a coequaliser of $a, b$ is an object $C$ and an arrow $c: B \rightarrow C$ such that $a c=b c$ and that $c$ is universal for this property. This means that if $d: B \rightarrow D$ is another arrow such that $a d=b d$, then there is a unique arrow $f: C \rightarrow D$ such that $c f=d$, as in (7). Again, a coequaliser is uniquely determined up to isomorphism.

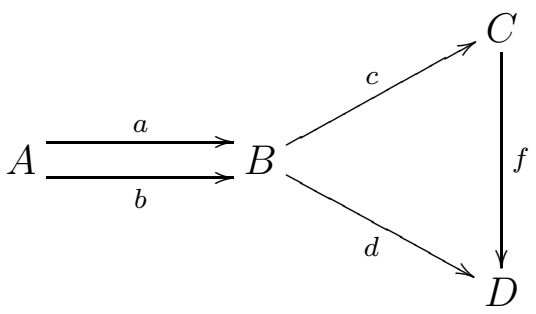

The product is a special case of the notion of limit, and the coequaliser is a special case of the notion of colimit. The latter notion is important when considering the intuitive notion of gluing pieces together. In particular, a digraph $D$ can be considered as a coequaliser of functions $s, t: U \rightarrow D$, where $U$ is a disjoint union of vertices, loops and arcs, and the functions $s, t$ describe how these elements are glued together in $D$. For more details see $[4, \S 8.2]$.

Functors are morphisms of categories, preserving source, target and composition, and we shall use the notion of an adjoint pair of functors. Given two functors $F: C \rightarrow D$, $G: \mathrm{D} \rightarrow \mathrm{C}$, we say $F$ is left adjoint to $G$ if there are natural bijections

$$
\phi: \mathrm{D}(F C, D) \rightarrow \mathrm{C}(C, G D)
$$

for all objects $C$ of $C$ and $D$ of D. It may then be proved that $G$ is determined up to natural equivalence by $F$, and vice versa. 
An important use of adjoints is that, when $F$ is left-adjoint to $G, F$ preserves colimits (and, in particular, coequalisers), and $G$ preserves limits (and, in particular, products). We give here the standard proof for the case of products.

Let $p_{1}: D \rightarrow A_{1}, p_{2}: D \rightarrow A_{2}$ be a product of $A_{1}, A_{2}$ in $\mathrm{D}$. Then we have natural bijections

$$
\begin{aligned}
\mathrm{C}(C, G D) & \cong \mathrm{D}(F C, D) \cong \mathrm{D}\left(F C, A_{1}\right) \times \mathrm{D}\left(F C, A_{2}\right) \\
& \cong \mathrm{C}\left(C, G A_{1}\right) \times \mathrm{C}\left(C, G A_{2}\right) \cong \mathrm{C}\left(C, G A_{1} \times G A_{2}\right)
\end{aligned}
$$

Now taking $C=G D$ we get in C a morphism $f: G D \rightarrow G A_{1} \times G A_{2}$, and taking $C=G A_{1} \times G A_{2}$ we get a morphism $g: G A_{1} \times G A_{2} \rightarrow G D$. It is then easy to prove, using naturality of the bijections, that $f g=$ id and $g f=$ id.

Suppose now that $\mathrm{C}$ is a category with finite products. We say that $\mathrm{C}$ is closed if, for all objects $A$ of $\mathrm{C}$, the functor $-\times B: \mathrm{C} \rightarrow \mathrm{C}$ has a right adjoint. This is equivalent to saying that, for all objects $B, C$ of $\mathrm{C}$, there is an object $\operatorname{HOM}(B, C)$ of $\mathrm{C}$, functorial in $B, C$, together with natural bijections

$$
\mathrm{C}(A \times B, C) \cong \mathrm{C}(A, \operatorname{HOM}(B, C))
$$

for all objects $A, B, C$ of $\mathrm{C}$. The object $\operatorname{HOM}(B, C)$ is called the internal hom in $\mathrm{C}$.

A category $\mathrm{C}$ is cartesian closed if it has a terminal object; all finite products specified; and internal homs. These functors are right adjoint to $0: \mathrm{C} \rightarrow 1$; the diagonal functor $\mathrm{C} \rightarrow \mathrm{C} \times \mathrm{C}$; and $(-\times B): \mathrm{C} \rightarrow \mathrm{C}$ respectively.

A topos is a category with properties analogous to those of the category Set of sets and functions. Specifically, it has finite limits and colimits, is cartesian closed, and has a subobject classifier (see [18], I.6).

An example of a cartesian closed category is the category Cat of small categories and functors. The internal hom in this category is written CAT. The objects of CAT(C, D) are the functors $C \rightarrow D$ and the morphisms are the natural transformations of these functors. In fact CAT (C, D) is a well defined category as long as $C$ is small.

One of the applications of these results is that, for any cartesian closed category of graphs, since a product of graphs is symmetric the product $G \times H$ can be built up from knowing the product of the basic elements of $G$ and $H$, namely loops, bands and edges.

An important result is that if $\mathrm{C}$ is a small category, and $\mathrm{C}^{\mathrm{op}}$ is the category opposite to $\mathrm{C}$, then the functor category $\mathrm{CAT}\left(\mathrm{C}^{\mathrm{op}}\right.$, Set $)=\mathrm{Set}^{\mathrm{C}^{\mathrm{op}}}$ is a topos (see for example [18]). Now Dgph $=\operatorname{CAT}\left(\mathrm{C}_{3}^{\text {op }}\right.$, Set $)$ where $\mathrm{C}_{3}$ is the category with one object and three arrows,

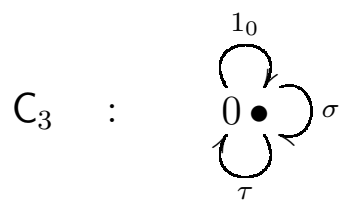


and composition rules $\sigma \tau=\sigma, \tau \sigma=\tau$, from which it follows that $\sigma^{2}=\sigma, \tau^{2}=\tau$. This means that $D \in$ Dgph may be considered as the functor $\mathcal{D}: \mathrm{C}_{3}^{\text {op }} \rightarrow$ Set in which

$$
\mathcal{D}(0)=U(D), \quad \mathcal{D}\left(1_{0}\right)=1_{U(D)}, \quad \mathcal{D}(\sigma)=s, \quad \mathcal{D}(\tau)=t
$$

Since $\mathrm{C}_{3}$ has a single object it is a monoid, and we say that Dgph is a category of actions of $\mathrm{C}_{3}$. Similarly $\mathrm{Tdgph}=\mathrm{CAT}\left(\mathrm{C}_{4}^{\mathrm{op}}\right.$, Set $)$ where $\mathrm{C}_{4}$ has objects 1,2 ; two identity arrows; and $\mathrm{C}_{4}(1,2)=\{\sigma, \tau\}$, (see $\left.[7]\right)$ :

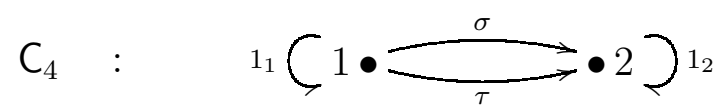

In what follows we assume that all graphs and digraphs are reflexive unless otherwise stated.

\section{The category of reflexive, directed graphs}

The simplest digraph is the empty digraph $\emptyset$, and the next simplest is the terminal digraph $T$ with only one element, which is necessarily a vertex. For every digraph $D$ both $\operatorname{Dgph}(\emptyset, D)$ and $\operatorname{Dgph}(D, T)$ are singletons, while $\operatorname{Dgph}(T, D)$ is bijective with the set of vertices of $D$.

There is a standard 1-arc digraph $I$ and a 1-loop digraph $\Lambda$ pictured as:

$$
I: \quad 1 \bullet \stackrel{\iota}{\longrightarrow} \bullet 2 \quad \Lambda: \quad 0 \bullet\rangle
$$

The digraph $I$ plays a special role in Dgph, namely that $L(D) \cup A(D)$ is bijective with the set of morphisms $I \rightarrow D$. Further, given two morphisms $\mu, \nu: D \rightarrow D^{\prime}$, we have $\mu=\nu$ if and only if $\mu \circ \pi=\nu \circ \pi$ for all morphisms $\pi: I \rightarrow D$. Thus $I$ is a generator for Dgph. This property is analogous to the property of the group of integers in the category of groups. We can also determine the loops in a digraph $D$ as the morphisms $\Lambda \rightarrow D$.

The properties of the category Dgph which we find most useful are the existence of products and of internal hom, the latter giving morphism digraphs.

The usual representation of the product of digraphs $D_{1}$ and $D_{2}$ is the digraph $D_{1} \times D_{2}$ with elements $U\left(D_{1}\right) \times U\left(D_{2}\right)$, source and target maps $s_{1} \times s_{2}$ and $t_{1} \times t_{2}$, and with the obvious projections from $D_{1} \times D_{2}$ to $D_{1}$ and $D_{2}$. Note that

$$
V\left(D_{1} \times D_{2}\right)=V\left(D_{1}\right) \times V\left(D_{2}\right) \quad \text { and } \quad L\left(D_{1} \times D_{2}\right)=L\left(D_{1}\right) \times L\left(D_{2}\right)
$$


We may display $I \times I$ and $I \times \Lambda$ as follows:
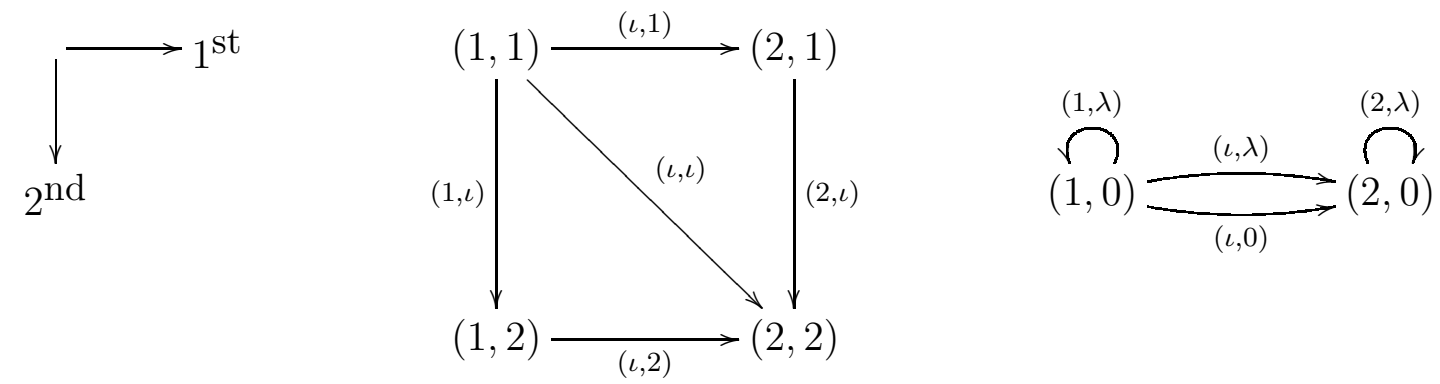

As another example, for any digraph $D$ there is a canonical isomorphism

$$
D \times T \rightarrow D, \quad(e, \bullet) \mapsto e .
$$

As discussed in the introduction, the internal hom $D^{C}=\operatorname{DGPH}(C, D)$ of two digraphs $C, D$ is a digraph defined by the condition that, for all digraphs $B$, there is a natural bijection

$$
\operatorname{Dgph}(B \times C, D) \cong \operatorname{Dgph}(B, \operatorname{DGPH}(C, D)) .
$$

This bijection characterises the digraph $H=\operatorname{DGPH}(C, D)$ up to isomorphism, by the uniqueness up to equivalence of right adjoints to a functor.

Equation (9) also allows us to prove the existence of $\operatorname{DGPH}(C, D)$, by identifying its vertices with the morphisms $T \rightarrow \operatorname{DGPH}(C, D)$, which are just the morphisms $C \rightarrow D$, and by identifying its loops and arcs with the morphisms $I \rightarrow \operatorname{DGPH}(C, D)$. Thus the loops or arcs $\operatorname{DGPH}(C, D)\left(\nu_{1}, \nu_{2}\right)$ are morphisms of the form

$$
\psi^{\prime} \quad: \quad I \times C \rightarrow D, \quad(1, a) \mapsto \nu_{1} a, \quad(2, a) \mapsto \nu_{2} a, \quad(\iota, a) \mapsto \psi a \in D\left(\nu_{1} s a, \nu_{2} t a\right) .
$$

For the diagram form of the internal hom we identify $\left(\psi^{\prime}: \nu_{1} \longrightarrow \nu_{2}\right)$ with the function

$$
\psi: U(C) \rightarrow U(D), \quad(a: u \longrightarrow v) \mapsto\left(\psi a: \nu_{1} u \longrightarrow \nu_{2} v\right)
$$

so that each arc of $\operatorname{DGPH}(C, D)$ is a 'diagram of type $C$ in $D$ '.

Example 3.1 If $1_{A}$ is the identity morphism, and $\rho$ the other automorphism of the digraph $A$ shown below, there are arrows $\left(\psi: 1_{A} \longrightarrow \rho\right)$ and $\left(\phi: \rho \longrightarrow 1_{A}\right)$ in $\operatorname{DGPH}(A, A)$ :

$$
A: \quad 0 \stackrel{a}{b} 1 \quad \operatorname{DGPH}(A, A):
$$

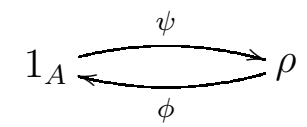

and $\operatorname{DGPH}(A, A)$ has diagram form

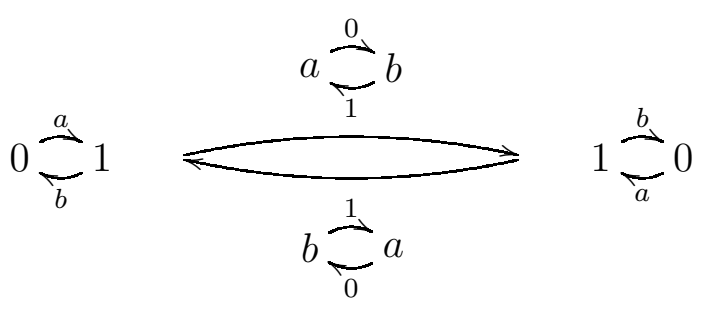


Either of these explicit descriptions can now be used as a definition of the morphism digraph $\operatorname{DGPH}(C, D)$, and the exponential law (9) may be verified directly. As we shall explain later (see the proof of Proposition 6.2) this law is a special case of results on functor categories, and so we do not give a proof here.

Remark 3.2 An alternative, non-categorical version of an exponential digraph $D^{C}$ is frequently used in articles on Hedetniemi's conjecture, which states that $\chi(G \times H)=$ $\min \{\chi(G), \chi(H)\}$. It was shown in [8] that this conjecture is equivalent to the statement that $K_{k}^{G}$ is $k$-colourable whenever $\chi(G)>k$. In these articles the digraph $D^{C}$ has as vertices the morphisms $C \rightarrow D$ and there is an arc $f \rightarrow g$ provided there is an arc $f(x) \rightarrow g(y)$ in $D$ whenever there is an arc $x \rightarrow y$ in $C$. Such a $D^{C}$ is necessarily relational (strict??), which makes sense for vertex colourings, whereas our $D^{C}$ need not be. The corresponding definition of $H^{G}$ for graphs specifies an edge joining $f$ and $g$ provided $f(x)$ and $g(y)$ are adjacent in $H$ whenever $x, y$ are adjacent in $G$. The survey [20] by Sauer, and the references therein, give further details.

In the language of category theory, the category Dgph is said to be cartesian closed, since all finite products exist and the functor $(-) \times C$ is defined for every digraph $C$. There are a number of useful properties of cartesian closed categories which we can exploit, and which can be interpreted in terms of our explicit description of the morphism digraph. First, there is an evaluation morphism

$$
\varepsilon_{C, D}: \operatorname{DGPH}(C, D) \times C \rightarrow D, \quad\left(\psi^{\prime}, a\right) \mapsto \psi^{\prime}(\iota, a)=\psi a
$$

Second, there is a composition morphism of digraphs

$$
\gamma_{B, C, D}: \operatorname{DGPH}(C, D) \times \operatorname{DGPH}(B, C) \rightarrow \operatorname{DGPH}(B, D)
$$

under which

$$
\gamma_{B, C, D}\left(\psi^{\prime}, \phi^{\prime}\right): I \times B \stackrel{\delta \times 1}{\longrightarrow} I \times I \times B \stackrel{1 \times \phi^{\prime}}{\longrightarrow} I \times C \stackrel{\psi^{\prime}}{\longrightarrow} D,
$$

where $\delta: I \rightarrow I \times I$ is the diagonal morphism. When there is no need to refer to $B, C, D$ explicitly, we simply write $\gamma\left(\psi^{\prime}, \phi^{\prime}\right)$. In terms of the diagram form we have

$$
\left(\psi: \nu_{1} \longrightarrow \nu_{2}\right) \circ\left(\phi: \mu_{1} \longrightarrow \mu_{2}\right)=\left(\psi \circ \phi: \nu_{1} \circ \mu_{1} \longrightarrow \nu_{2} \circ \mu_{2}\right): U(B) \rightarrow U(D)
$$

Note that $\gamma_{B, C, D}$ is adjoint to the composite

$$
\operatorname{DGPH}(C, D) \times \operatorname{DGPH}(B, C) \times B \quad \stackrel{1 \times \varepsilon_{B, C}}{\longrightarrow} \operatorname{DGPH}(C, D) \times C \stackrel{\varepsilon_{C, D}}{\longrightarrow} D .
$$

For a given digraph $D$, the digraph $\operatorname{DGPH}(D, D)$ with composition $\gamma_{D, D, D}$ forms the monoid digraph $\operatorname{END}(D)$, which has the structure of both a digraph and a monoid. That 
is, the monoid structure $\operatorname{END}(D) \times \operatorname{END}(D) \rightarrow \operatorname{END}(D)$ is a morphism of digraphs. Any monoid in Set has a maximal subgroup. This is equally true in the category Dgph, where the maximal subgroup of $\operatorname{END}(D)$ is written $\operatorname{AUT}(D)$ and called the group digraph of $D$. In terms of the diagram form, $\operatorname{AUT}(D)$ consists of those $\left.\left(\psi: \nu_{1} \longrightarrow \nu_{2}\right)\right)$, such that each of $\psi, \nu_{1}, \nu_{2}$ is a bijection. It is this group digraph which is studied by Shrimpton in $[22,23]$. We now give some examples of morphism digraphs, and later examine an analogue for undirected graphs.

The subcategory Sdgph of strict digraphs is closed under products and, since there is at most one choice of $\psi$ in equation (10), closed under internal homs. Since it is not possible in $\operatorname{END}(D)$ to have arrows $\left(\psi: \nu_{1} \longrightarrow \nu_{2}\right),\left(\phi: \mu_{1} \longrightarrow \mu_{2}\right)$ with $\psi=\phi$ but not $\nu_{1}=\mu_{1}$ and $\nu_{2}=\mu_{2}$, the monoid has a faithful representation as a transformation monoid on $U(D)$.

\section{$4 \quad$ Examples}

We now include a collection of examples which illustrate the main features of morphism digraphs.

Example 4.1 For any digraph $D$ there is a canonical isomorphism $D \rightarrow \operatorname{DGPH}(T, D)$ given by

$$
(a: u \longrightarrow v) \mapsto \begin{cases}\psi_{a}^{\prime}: I \times T \rightarrow D, & (\iota, \bullet) \mapsto a \\ \psi_{a}: U(T) \rightarrow U(D), & \bullet \mapsto a .\end{cases}
$$

Example 4.2 For any digraph $D$, the vertices of $\operatorname{DGPH}(I, D)$ are bijective with the loops and $\operatorname{arcs}$ of $D$. Suppose $(c: u \longrightarrow v),(d: x \longrightarrow y)$ in $D$. Then an $\operatorname{arc}\left(\psi^{\prime}: c \longrightarrow d\right)$ of $\operatorname{DGPH}(I, D)$ is a morphism $\psi^{\prime}: I \times I \longrightarrow D$ such that $\psi^{\prime}(1, \iota)=c, \psi^{\prime}(2, \iota)=d$, and so is specified by a diagram in $D$ of the type

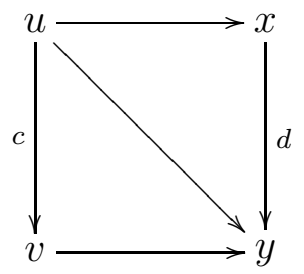

Hence the number of loops and arcs in $\operatorname{DGPH}(I, D)(c, d)$ is the product of the cardinalities of $D(u, x), D(u, y)$ and $D(v, y)$. In particular, $\operatorname{DGPH}(I, I)$ is the digraph

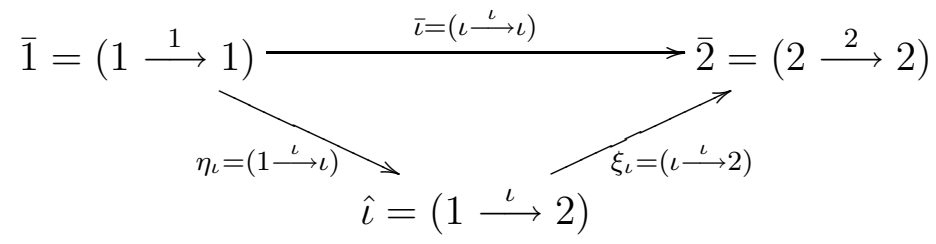


(see Lawvere ([15], p.276) for an interpretation using processes).

Notice that $\operatorname{DGPH}(I, D)$ contains a set of constant functions $\bar{e}$, forming an isomorphic copy $\bar{D}$ of $D$, where

$$
U(\bar{D})=\{\bar{e}: U(I) \rightarrow U(D) \mid e \in U(D), \bar{e}(1)=\bar{e}(2)=\bar{e}(\iota)=e\} .
$$

Also, for each loop or arc $(e: u \longrightarrow v)$ in $D$, the digraph $\operatorname{DGPH}(I, D)$ contains arcs $\hat{e}=(u \stackrel{e}{\longrightarrow} v), \eta_{e}=(u \stackrel{e}{\longrightarrow} e), \xi_{e}=(e \stackrel{e}{\longrightarrow} v)$, and the path

$$
\bar{u} \stackrel{\eta_{e}}{\longrightarrow} \hat{e} \stackrel{\xi_{e}}{\longrightarrow} \bar{v} .
$$

The evaluation morphism $\varepsilon_{I, I}$, defined by (11), restricts to

$$
\varepsilon_{1}=\varepsilon_{I, I}(-, 1), \quad \varepsilon_{2}=\varepsilon_{I, I}(-, 2) \quad: \quad \operatorname{DGPH}(I, I) \rightarrow I,
$$

both of which map $\bar{\iota}$ to $\iota$, while $\varepsilon_{1}\left(\eta_{\iota}\right)=1, \varepsilon_{1}\left(\xi_{\iota}\right)=\iota$, and $\varepsilon_{2}\left(\eta_{\iota}\right)=\iota, \varepsilon_{2}\left(\xi_{\iota}\right)=2$.

Under the composition defined in (12), (13) the monoid digraph $\operatorname{END}(I)$ has identity $\hat{\imath}$ and multiplication table

\begin{tabular}{|c|cccccc|}
\hline & $\hat{\iota}$ & $\overline{1}$ & $\overline{2}$ & $\bar{\iota}$ & $\eta_{\iota}$ & $\xi_{\iota}$ \\
\hline$\hat{\iota}$ & $\hat{\iota}$ & $\overline{1}$ & $\overline{2}$ & $\bar{\iota}$ & $\eta_{\iota}$ & $\xi_{\iota}$ \\
$\overline{1}$ & $\overline{1}$ & $\overline{1}$ & $\overline{1}$ & $\overline{1}$ & $\overline{1}$ & $\overline{1}$ \\
$\overline{2}$ & $\overline{2}$ & $\overline{2}$ & $\overline{2}$ & $\overline{2}$ & $\overline{2}$ & $\overline{2}$ \\
$\bar{\iota}$ & $\bar{\iota}$ & $\bar{\iota}$ & $\bar{\iota}$ & $\bar{\iota}$ & $\bar{\iota}$ & $\bar{\iota}$ \\
$\eta_{\iota}$ & $\eta_{\iota}$ & $\overline{1}$ & $\bar{\iota}$ & $\bar{\iota}$ & $\eta_{\iota}$ & $\bar{\iota}$ \\
$\xi_{\iota}$ & $\xi_{\iota}$ & $\bar{\iota}$ & $\overline{2}$ & $\bar{\iota}$ & $\bar{\iota}$ & $\xi_{\iota}$ \\
\hline
\end{tabular}

Example 4.3 Let $P_{2}$ be the path digraph $u_{0} \stackrel{a_{1}}{\longrightarrow} u_{1} \stackrel{a_{2}}{\longrightarrow} u_{2}$. Since there is no arc from $u_{0}$ to $u_{2}$ in $P_{2}$, there is no arc from $\hat{a}_{1}$ to $\hat{a}_{2}$ in $\operatorname{DGPH}\left(I, P_{2}\right)$ :

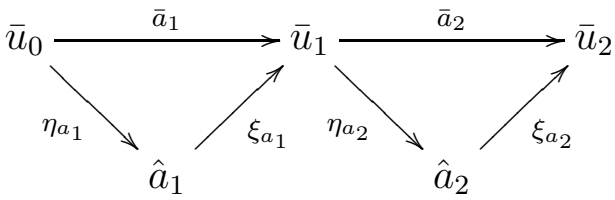

Similarly, if $P_{n}$ is the path digraph with vertices $\left\{u_{i} \mid 0 \leqslant i \leqslant n\right\}$ and $\operatorname{arcs}\left\{a_{j}=\left(u_{j-1} \longrightarrow\right.\right.$ $\left.\left.u_{j}\right) \mid 1 \leqslant j \leqslant n\right\}$ then $\operatorname{DGPH}\left(I, P_{n}\right)$ has $(2 n+1)$ vertices $\left\{\bar{u}_{i}, \hat{a}_{j}\right\}$ and $3 n \operatorname{arcs}\left\{\bar{a}_{j}, \eta_{a_{j}}, \xi_{a_{j}}\right\}$.

The digraph $\operatorname{DGPH}\left(P_{n}, I\right)$ has $(n+2)$ vertices: $\overline{1}, \overline{2}$ and, for $1 \leqslant i \leqslant n, \mu_{i}: P_{n} \rightarrow I$ such that $\mu_{i} a_{i}=\iota$. In this digraph there is a unique $\operatorname{arc}\left(\mu_{j} \longrightarrow \mu_{i}\right)$ for each $j>i$. The following diagram exhibits an $\operatorname{arc}\left(\mu_{5} \longrightarrow \mu_{2}\right) \in \operatorname{DGPH}\left(P_{7}, I\right)$ :

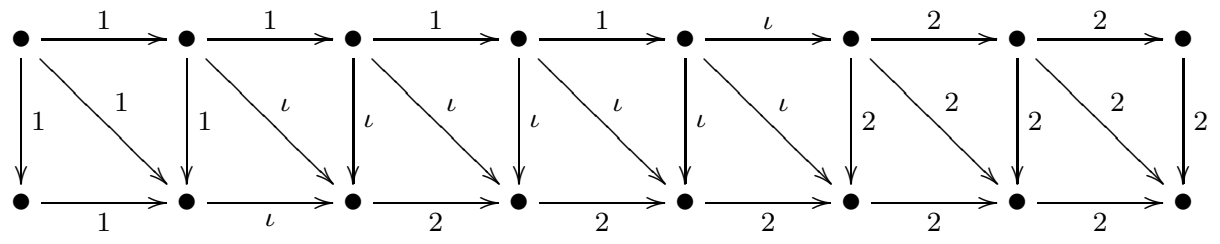


There is a unique arc from $\overline{1}$ to every vertex and also from every vertex to $\overline{2}$, giving a total of $\left(\begin{array}{c}n+3 \\ 2\end{array}\right)$ elements in $\operatorname{DGPH}\left(P_{n}, I\right)$, including $\left(\begin{array}{c}n+2 \\ 2\end{array}\right)$ arcs.

Example 4.4 Suppose now that $C_{3}$ is the circular digraph

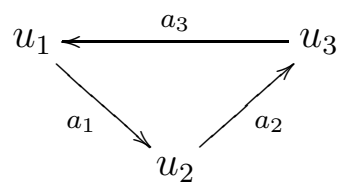

Then $\operatorname{DGPH}\left(I, C_{3}\right)$ is a digraph with six vertices, one for each element of $C_{3}$, having the form:

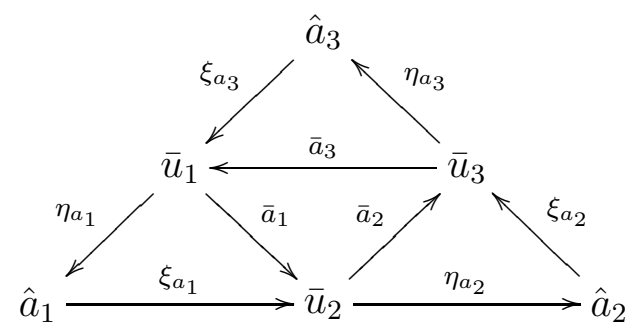

It is easy to see that, when $C_{n}$ is the circular digraph with $n \geqslant 4$ vertices $u_{i}$, and $n$ arcs $\left(a_{i}: u_{i} \longrightarrow u_{i+1}\right),\left(a_{n}: u_{n} \longrightarrow u_{1}\right)$, then $\operatorname{DGPH}\left(I, C_{n}\right)$ has $5 n$ elements $\left\{\bar{u}_{i}, \bar{a}_{i}, \hat{a}_{i}, \eta_{a_{i}}, \xi_{a_{i}}\right\}$.

The automorphisms of $C_{3}$ form the permutation group

$$
\left\{(), \pi=\left(u_{1} u_{2} u_{3}\right)\left(a_{1} a_{2} a_{3}\right), \pi^{2}=\left(u_{1} u_{3} u_{2}\right)\left(a_{1} a_{3} a_{2}\right)\right\}
$$

and there are three constant endomorphisms. There is no arrow $(\psi:() \longrightarrow \pi)$ since, in particular, there is no arrow in $C_{3}\left(u_{1}, u_{3}\right)$ to take as $\psi\left(a_{1}\right)$. So $\operatorname{AUT}\left(C_{3}\right)$ is discrete, and $\operatorname{END}\left(C_{3}\right)$ is the digraph

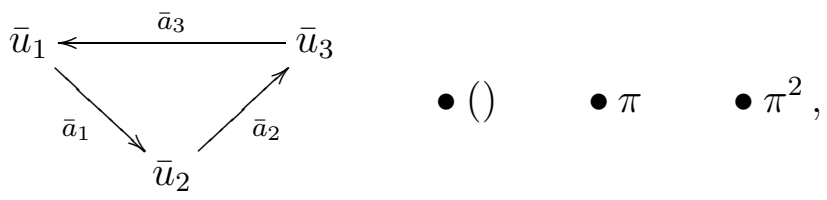

the monoid comprising three permutations and six left zeroes. Similarly $\operatorname{END}\left(C_{n}\right)$ contains the $2 n$ constant functions in $\bar{C}_{n}$ and $n$ isolated automorphisms.

Example 4.5 Suppose now that $S_{2}$ is the simplicial digraph

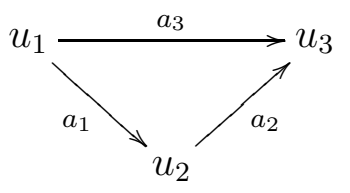


Then $\operatorname{DGPH}\left(I, S_{2}\right)$ has one more arc than $\operatorname{DGPH}\left(I, C_{3}\right)$ :

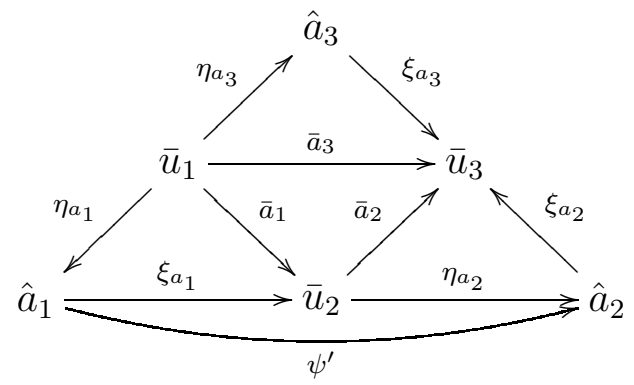

where $\psi^{\prime}: I \times I \rightarrow S_{2}$ and $\psi: U(I) \rightarrow U\left(S_{2}\right)$ may be pictured as:

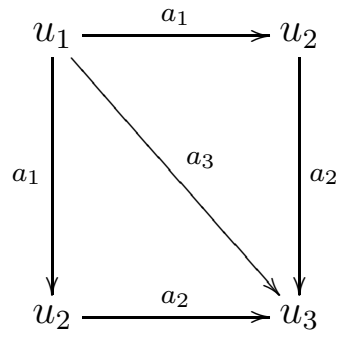

and

$$
\left(u_{1} \stackrel{a_{1}}{\longrightarrow} u_{2}\right) \stackrel{\left(a_{1} \stackrel{a_{3}}{\longrightarrow} a_{2}\right)}{\longrightarrow}\left(u_{2} \stackrel{a_{2}}{\longrightarrow} u_{3}\right) .
$$

Example 4.6 Denote by $K_{n}^{k}$ the $k$-complete digraph with $n$ vertices, $k-1$ additional loops at each vertex and, for each pair of distinct vertices $u$ and $v$, exactly $k$ arcs with source $u$ and target $v$. By previous results, $\operatorname{DGPH}\left(I, K_{n}^{k}\right)$ has as vertices the arcs of $K_{n}^{k}$ and there are $k^{3}$ arcs between any two of these vertices. Thus $\operatorname{DGPH}\left(I, K_{n}^{k}\right)$ is isomorphic to $K_{k n^{2}}^{k^{3}}$.

Example 4.7 An important example is the digraph

$$
\Omega:
$$

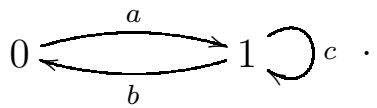

For any digraph $D$ the morphisms $\chi \in \operatorname{Dgph}(D, \Omega)$ may be identified with the subdigraphs of $D$ by $\chi \mapsto \chi^{-1}(1)$. The reason is that a subdigraph $C$ of $D$ has a characteristic morphism $\chi_{C}: D \rightarrow \Omega$ which maps all the vertices in $C$ to 1 ; the remaining vertices to 0 ; all the loops and arcs in $C$ to $c$; and the remaining elements to the only possible choice from $\{0,1, a, b\}$. Because of this property, the digraph $\Omega$ is a subobject classifier for directed digraphs. The lattice $\operatorname{Dgph}(\Omega, \Omega)$ of subdigraphs of $\Omega$ is not boolean since, in particular, the complement of the complement of $\{1\}$ is $\{1, c\}$. This lattice is an example of a Heyting algebra ([18], I.8). The existence of this subobject classifier is one of the reasons why digraphs may be considered as analogous to sets.

Taking $D=\Omega$, we summarise the 12 subgraphs and the corresponding endomorphisms of $\Omega$ in the following table: 


\begin{tabular}{|c|c|ccccc|}
\hline subgraph $C$ & morphism $\chi_{C}$ & $\chi_{C}(0)$ & $\chi_{C}(1)$ & $\chi_{C}(a)$ & $\chi_{C}(b)$ & $\chi_{C}(c)$ \\
\hline$\emptyset$ & $\chi_{1}$ & 0 & 0 & 0 & 0 & 0 \\
$\{0\}$ & $\chi_{2}$ & 1 & 0 & $b$ & $a$ & 0 \\
$\{1\},\{1, c\}$ & $\chi_{3}, \chi_{4}$ & 0 & 1 & $a$ & $b$ & $1 \mid c$ \\
$\{0,1\}, \ldots, \Omega$ & $\chi_{5}, \ldots, \chi_{12}$ & 1 & 1 & $1 \mid c$ & $1 \mid c$ & $1 \mid c$ \\
\hline
\end{tabular}

The digraph $\operatorname{END}(\Omega)$ has a total of 2417 arcs since the number of arcs from $\chi_{i}$ to $\chi_{j}$ is $2^{m_{i, j}}$ where $m_{i, j}$ is the number of arcs $e$ in $U(\Omega)$ such that $s \chi_{i} e=t \chi_{j} e=1$.

Since $\Omega$ has a loop $c$ it is not strict, so Sdgph has no subobject classifier and is not a topos.

Remark 4.8 One can use known properties of cartesian closed categories to analyse other constructions. For example, there is a natural isomorphism

$$
\left(D_{1} \times D_{2}\right)^{C} \cong D_{1}^{C} \times D_{2}^{C}
$$

More generally, if $D$ is given as a limit $D=\lim _{\lambda} D_{\lambda}$, then $\operatorname{DGPH}(C, D)$ is isomorphic to the $\operatorname{limit}_{\lim _{\lambda}}\left(D_{\lambda}^{C}\right)$. In the other direction, if $C$ is given as a colimit, $C=\operatorname{colim}_{\lambda} C_{\lambda}$, then $B \times C$ is isomorphic to $\operatorname{colim}_{\lambda}\left(B \times C_{\lambda}\right)$, and $\operatorname{DGPH}(C, D)$ is isomorphic to $\lim _{\lambda}\left(D^{C_{\lambda}}\right)$. These results can be practical tools for computation of examples of product and morphism digraphs.

\section{Undirected, reflexive graphs}

We now investigate analogues of the results in Section 3 for reflexive, undirected graphs. Recall that, for $X$ a set, we denote by $S^{2}(X)$ the quotient of $X \times X$ by the twist action $(x, y) \mapsto(y, x)$. A graph $G$ has an underlying set of elements $U(G)$. The source and target maps of directed graphs are replaced by a single boundary or end-points map $\partial: U(G) \rightarrow S^{2}(U(G))$ satisfying $\partial_{*} \circ \partial=\partial$. Then $V(G)=\{v \in G \mid \partial v=\{v\}\}$ is the set of vertices of $G$.

We denote an element $e$ by $(e: \partial e)$ when we wish to display its vertices. An edge of $G$ which is not a vertex but whose boundary is a singleton is a loop (we shall introduce bands in a moment). The remaining elements of $G$ are called edges. We denote the category of reflexive graphs with vertices, loops, and edges by Gph. A morphism $\mu: G \rightarrow G^{\prime}$ in Gph is a function $\mu: U(G) \rightarrow U\left(G^{\prime}\right)$ such that

$$
\partial^{\prime} \circ \mu=\mu_{*} \circ \partial: U(G) \rightarrow S^{2}\left(V\left(G^{\prime}\right)\right) .
$$

As discussed in Section 1, we need a larger category of undirected graphs containing bands. A band in $G$ is an element $b \in U(G)$, with singleton boundary $u$, pictured as 


\section{$u \supseteqq b$}

The set of bands is written $B(G)$. We denote the category of reflexive graphs with vertices, loops, bands, and edges by Ugph. A morphism $\mu$ in Ugph is again defined by (14), but now the image of an edge may be an edge, a band, a loop, or a vertex. Similarly the image of a band may be a band, a loop or a vertex, but a loop may not be mapped to a band.

The product in Gph and in Ugph is defined by the universal property (5). We prove its existence, first in Gph and then in Ugph, by a direct construction. For $G_{1}, G_{2} \in \mathrm{Gph}$, the product graph $G_{1} \times G_{2}$ has vertex set $V\left(G_{1} \times G_{2}\right)=V\left(G_{1}\right) \times V\left(G_{2}\right)$ while the loops and edges of the product consist of all pairs

$$
\left(\left(e_{1}, e_{2}\right),\left\{\left(u_{1}, u_{2}\right),\left(v_{1}, v_{2}\right)\right\}\right)
$$

where

$$
\partial e_{1}=\left\{u_{1}, v_{1}\right\}, \quad \partial e_{2}=\left\{u_{2}, v_{2}\right\}, \quad \partial\left(\left(e_{1}, e_{2}\right),\left\{\left(u_{1}, u_{2}\right),\left(v_{1}, v_{2}\right)\right\}\right)=\left\{\left(u_{1}, u_{2}\right),\left(v_{1}, v_{2}\right)\right\} .
$$

For example,

$$
u_{1} e_{1} v_{1} \supset \ell \times u_{2} \frac{e_{2}}{v_{2}}=v_{\left(u_{1}, e_{2}\right)}^{\left(u_{1}, u_{2}\right)}
$$

The projections $p_{1}, p_{2}$ from the product to $G_{1}$ and $G_{2}$ send $\left(\left(e_{1}, e_{2}\right),\left\{\left(u_{1}, u_{2}\right),\left(v_{1}, v_{2}\right)\right\}\right)$ to $e_{1}$ and $e_{2}$ respectively. This implies that if $e_{1}$ and $e_{2}$ are both edges, then the product contains a second edge $\left(\left(e_{1}, e_{2}\right),\left\{\left(u_{1}, v_{2}\right),\left(v_{1}, u_{2}\right)\right\}\right)$ which also projects to $e_{1}$ and $e_{2}$. The universal property follows from the construction because, if $\mu_{1}: G_{1} \rightarrow G_{1}^{\prime}, \mu_{2}: G_{2} \rightarrow G_{2}^{\prime}$ are morphisms, then the morphism $\mu: G_{1} \times G_{2} \rightarrow G_{1}^{\prime} \times G_{2}^{\prime}$, with components $\mu_{1}$ and $\mu_{2}$, acts as $\mu_{1} \times \mu_{2}$ on vertices, and sends an edge $e$ with boundary $\{u, v\}$ to the edge

$$
\left(\left(\mu_{1} e, \mu_{2} e\right),\left\{\left(\mu_{1} u, \mu_{2} u\right),\left(\mu_{1} v, \mu_{2} v\right)\right\}\right) .
$$

This is the only definition consistent with $\mu$ being a morphism with the required projections. Of course, a second edge of the product

$$
\left(\left(\mu_{1} e, \mu_{2} e\right),\left\{\left(\mu_{1} u, \mu_{2} v\right),\left(\mu_{1} v, \mu_{2} u\right)\right\}\right)
$$


also projects to $\mu_{1} e$ and $\mu_{2} e$, but it is not in general the value of a morphism on $e$.

The category Gph is not cartesian closed, and hence cannot be expressed in the form CAT $\left(B^{o p}\right.$, Set $)$. To demonstrate this, we show in the following counterexample that products do not preserve coequalisers, ([22, p.44]). The identification of vertices referred to in the introduction may be achieved using a coequaliser. For the categorical background of this example, we refer to [17, IV.3].

Counterexample 5.1 Let $A$ be the graph with a single vertex $x$ and let $B$ be the graph with two vertices $u, v$ and a single edge $e$ joining them. Define $\alpha, \beta: A \rightarrow B$ by $\alpha x=u$ and $\beta x=v$. The coequaliser of $\alpha, \beta$ in $\mathrm{Gph}$ is a pair $(C, \gamma)$ where $\gamma$ is a morphism $\gamma: B \rightarrow C$ such that $\alpha \gamma=\beta \gamma$, satisfying the universal property shown in the following diagram:

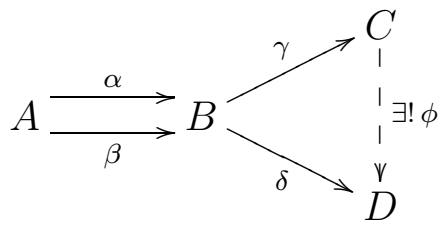

We may take $C$ to be the graph shown below, and $\gamma(e)=\ell$ :

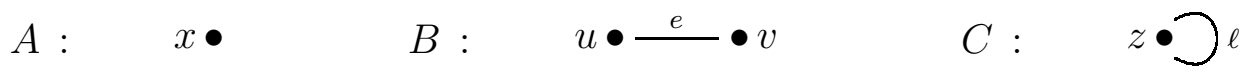

Consider now the two graphs:

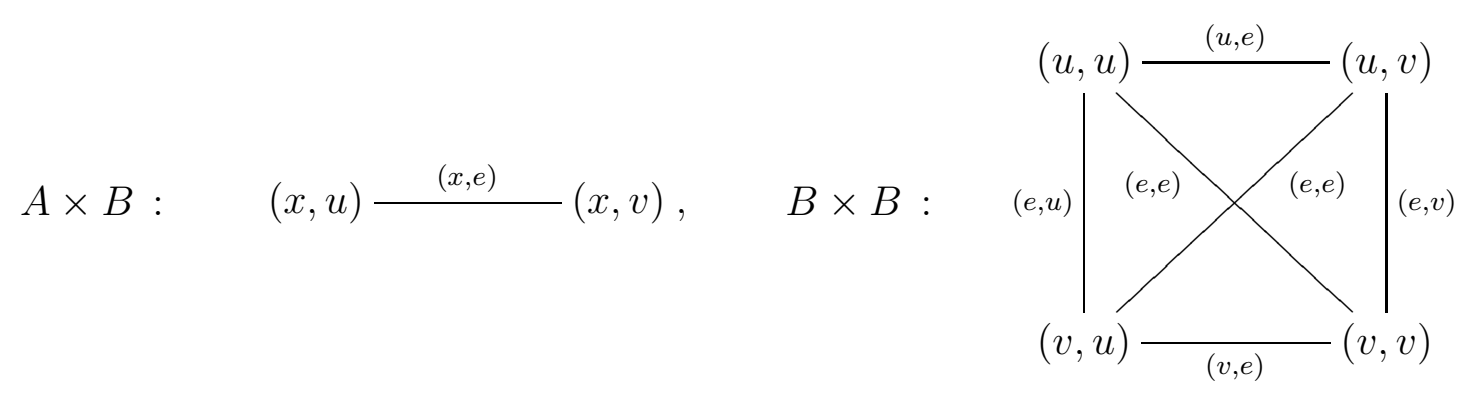

The graph $C \times B$ and the coequaliser of $\alpha \times 1_{B}, \beta \times 1_{B}: A \times B \rightarrow B \times B$ are pictured as follows, and are not isomorphic:
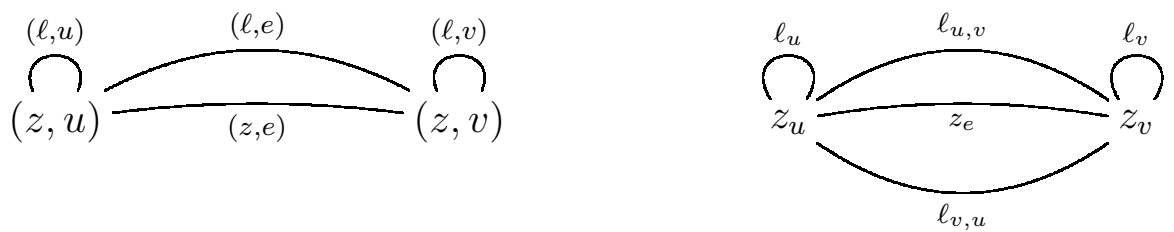

The construction of a product $B_{1} \times B_{2}$ in Ugph is the same as the construction in Gph, but now we must specify what happens with a band: 
- a loop or vertex in $B_{1}$ and a band in $B_{2}$ give rise to a band in the product;

- a band in $B_{1}$ and a band in $B_{2}$ give rise to two bands in the product;

- an edge in $B_{1}$ and a band in $B_{2}$ give rise to two edges in the product.

Then, in Ugph, the coequaliser $C^{\prime}$ of $\alpha, \beta$ and the product $C^{\prime} \times B$ are pictured as:

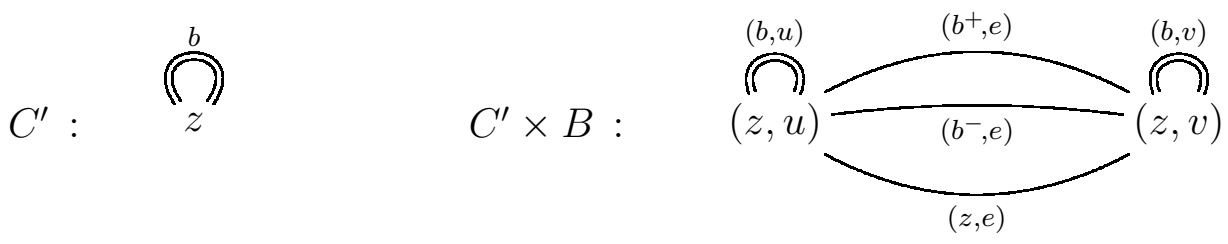

containing one and two bands respectively. Now $C^{\prime} \times B$ is isomorphic to the coequaliser of $\alpha \times 1_{B}$ and $\beta \times 1_{B}$ in Ugph.

\section{Reversible Digraphs}

One advantage of the cartesian closedness of Dgph $=\mathrm{CAT}\left(\mathrm{C}^{\mathrm{op}}\right.$, Set $)$, as in (8), is that the monoid digraph $\operatorname{END}(D)$ is an object in Dgph, and so is a digraph and also a monoid. This construction is not available for graphs. One solution to this difficulty is to embed Gph as a subcategory Rdgph of reversible digraphs in Dgph. Alternatively, we may choose to work with Ugph which is isomorphic to Rdgph. In either case, we wish to associate to a graph $G$ a reversible digraph $D$ (called a symmetric digraph in [20]) having endomorphism monoid isomorphic to that of $G$, calculate the monoid digraph of $D$, and derive from this an undirected graph as a candidate for the monoid graph of $G$.

We now give the formal definition of Rdgph. A reversal of a reflexive digraph $D$ is a direction-reversing, involutive endofunction $r$ on $U(D)$ satisfying:

$$
r^{2}=\mathrm{id}, \quad s \circ r=t, \quad t \circ r=s, \quad r \circ s=s, \quad r \circ t=t .
$$

We call $r a$ the reverse of $a$. Note that $r v=v$ for every vertex $v$. A loop $\ell$ in $D$ is called self-reverse if $r \ell=\ell$, otherwise $(\ell, r \ell)$ is a loop pair. A reversible digraph is a pair $(D, r)$ where $r$ is a reversal of $D$, and is pair-free if all loops are self-reverse. A morphism $\mu:(D, r) \rightarrow\left(D^{\prime}, r^{\prime}\right)$ of reversible digraphs is a digraph morphism $\mu: D \rightarrow D^{\prime}$ such that $\mu \circ r=r^{\prime} \circ \mu$. This gives a category Rdgph of reversible digraphs and their morphisms.

We need to consider how the categorical constructions for Dgph of Sections 3 and 4 transfer to Rdgph. The product of $\left(D_{1}, r_{1}\right) \times\left(D_{2}, r_{2}\right)$ is $\left(D_{1} \times D_{2}, r_{1} \times r_{2}\right)$. The subobject classifier $\Omega$ of Example 4.7 has just one reversal, with $r_{\Omega} a=b$ and $r_{\Omega} c=c$, and $\left(\Omega, r_{\Omega}\right)$ 
is a subobject classifier in Rdgph. Further, Rdgph has the form CAT(Fop , Set) where $F$ is the four-loop category with composition table shown below:

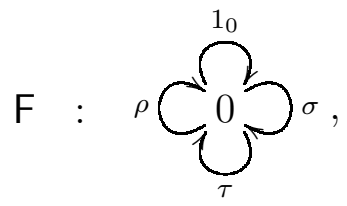

\begin{tabular}{|c|cccc|}
\hline$\circ$ & $1_{0}$ & $\sigma$ & $\tau$ & $\rho$ \\
\hline $1_{0}$ & $1_{0}$ & $\sigma$ & $\tau$ & $\rho$ \\
$\sigma$ & $\sigma$ & $\sigma$ & $\sigma$ & $\sigma$ \\
$\tau$ & $\tau$ & $\tau$ & $\tau$ & $\tau$ \\
$\rho$ & $\rho$ & $\tau$ & $\sigma$ & $1_{0}$ \\
\hline
\end{tabular}

As explained in Section 1, the category Rdgph is a category of actions of the monoid F, and so is a topos and, in particular, is cartesian closed.

Proposition 6.1 The reversible digraph corresponding to $\mathrm{F}(-, 0)$ under the isomorphism $\mathrm{Rdgph} \cong \mathrm{CAT}\left(\mathrm{F}^{\mathrm{op}}\right.$, Set $)$ is

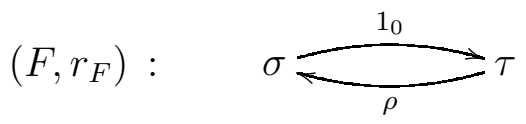

and is a generator for Rdgph.

Proof: The functor $\mathrm{F}(-, 0): \mathrm{F}^{\mathrm{op}} \rightarrow$ Set takes the single object 0 of $\mathrm{F}$ to the set $U(F)=\left\{1_{0}, \sigma, \tau, \rho\right\}$, and the arcs of $F$ to the following functions:

$$
\begin{aligned}
1_{0} & \mapsto 1_{F}:\left(1_{0}, \sigma, \tau, \rho\right) \mapsto\left(1_{0}, \sigma, \tau, \rho\right), \\
\sigma & \mapsto s_{F}:\left(1_{0}, \sigma, \tau, \rho\right) \mapsto(\sigma, \sigma, \tau, \tau), \\
\tau & \mapsto t_{F}:\left(1_{0}, \sigma, \tau, \rho\right) \mapsto(\tau, \sigma, \tau, \sigma), \\
\rho & \mapsto r_{F}:\left(1_{0}, \sigma, \tau, \rho\right) \mapsto\left(\rho, \sigma, \tau, 1_{0}\right)
\end{aligned}
$$

These functions determine the source, target and reverse of the arcs in $F$.

The arcs of a reversible digraph $(D, r)$ are bijective with the morphisms $\left(F, r_{F}\right) \rightarrow$ $(D, r)$ with $a \in U(D)$ corresponding to the reversal-preserving morphism which maps $1_{0}$ to $a$, so $\left(F, r_{F}\right)$ is a generator for Rdgph.

The internal hom RDGPH in Rdgph is described by the following result.

Proposition 6.2 The reversible morphism digraph $(R, \bar{r})=\operatorname{RDGPH}\left(\left(D_{1}, r_{1}\right),\left(D_{2}, r_{2}\right)\right)$ is the full subdigraph of $\mathrm{DGPH}\left(D_{1}, D_{2}\right)$ on the vertices corresponding to reversal-preserving morphisms. The reversal $\bar{r}$ on $R$ is given by:

$$
\bar{r}(\psi: \mu \longrightarrow \nu)=(\chi: \nu \longrightarrow \mu) \text { where } \chi(a)=r_{2} \psi r_{1}(a) \text { for all } a \in U\left(D_{1}\right) \text {. }
$$

Proof: By the Yoneda lemma, and the fact that $(-)^{D_{1}}$ is right adjoint to $(-) \times D_{1}$, the functor $\mathcal{D}=\operatorname{DGPH}\left(D_{1}, D_{2}\right): \mathrm{F}^{\text {op }} \rightarrow$ Set acts on $x \in \mathrm{Obj}(\mathrm{F})$ by

$$
\mathcal{D} x \cong \operatorname{Nat}\left(\mathrm{F}(-, x) \times D_{1}, D_{2}\right),
$$


and on an $\operatorname{arc} \alpha \in \mathrm{F}(-, x)$ by

$$
\mathcal{D} \alpha(f)=f \circ(\mathrm{F}(-, \alpha) \times 1) \text { where } f \in \operatorname{DGPH}\left(D_{1}, D_{2}\right)(x) .
$$

Taking $\mathrm{F}(-, 0)=\left(F, r_{F}\right)$ as above, the arcs of $F \times D_{1}$ are given by

$$
\left\{\left(1_{0}, a\right),(\sigma, a),(\tau, a),(\rho, a) \mid a \in U\left(D_{1}\right)\right\},
$$

with the vertices being

$$
\left\{(\sigma, u),(\tau, u) \mid u \in V\left(D_{1}\right)\right\} .
$$

Thus a morphism $\Psi \in \operatorname{Dgph}\left(F \times D_{1}, D_{2}\right)$ is a 4 -tuple of functions $(\psi, \mu, \nu, \theta)$ : $U\left(D_{1}\right) \rightarrow U\left(D_{2}\right)$ where

$$
\Psi\left(1_{0}, a\right)=\psi(a), \quad \Psi(\sigma, a)=\mu(a), \quad \Psi(\tau, a)=\nu(a), \quad \Psi(\rho, a)=\theta(a) .
$$

Then for any $a \in U\left(D_{1}\right)$, and $q \in\{s, t, r\}$,

$$
q_{2}(\mu a)=q_{2}(\Psi(\sigma, a))=\Psi\left(\sigma, q_{1} a\right)=\mu\left(q_{1} a\right),
$$

and similarly for $\nu$. Hence $\mu, \nu$ are both morphisms $\left(D_{1}, r_{1}\right) \rightarrow\left(D_{2}, r_{2}\right)$.

On the other hand,

$$
\begin{aligned}
& s_{2}(\psi a)=s_{2}\left(\Psi\left(1_{0}, a\right)\right)=\Psi\left(\sigma, s_{1} a\right)=\mu\left(s_{1} a\right), \\
& t_{2}(\psi a)=t_{2}\left(\Psi\left(1_{0}, a\right)\right)=\Psi\left(\tau, t_{1} a\right)=\nu\left(t_{1} a\right), \\
& r_{2}(\psi a)=r_{2}\left(\Psi\left(1_{0}, a\right)\right)=\Psi\left(\rho, r_{1} a\right)=\theta\left(r_{1} a\right),
\end{aligned}
$$

which implies

$$
s_{2} \circ \psi=\mu \circ s_{1}, \quad t_{2} \circ \psi=\nu \circ t_{1}, \quad \theta=r_{2} \circ \psi \circ r_{1} .
$$

Thus $\theta$ is completely determined by $\psi$.

Also, for any $\beta \in \mathrm{F}(0,0), a \in U\left(D_{1}\right)$,

$$
s_{2}(\Psi(\beta, a))=\operatorname{DGPH}\left(D_{1}, D_{2}\right)(\sigma) . \Psi(\beta, a)=\Psi \circ(\mathrm{F}(0, \sigma) \times 1)(\beta, a)=\Psi(\sigma \beta, a)=\mu a
$$

since $\sigma 1_{0}=\sigma^{2}=\sigma \tau=\sigma$. So $s(\psi: \mu \longrightarrow \nu)=\mu$ and similarly $t(\psi: \mu \longrightarrow \nu)=\nu$ and $r(\psi: \mu \longrightarrow \nu)=(r \psi r: \nu \longrightarrow \mu)$. This means that $\operatorname{RDGPH}\left(D_{1}, D_{2}\right)$ is simply the full subgraph of $\operatorname{DGPH}\left(D_{1}, D_{2}\right)$ on the vertices corresponding to reversal-preserving morphisms.

Corollary 6.3 The reversal $\bar{r}$ on the monoid digraph $\operatorname{END}(D, r)$ preserves the monoid multiplication.

Proof: Given $\left(\psi: \nu_{1} \longrightarrow \nu_{2}\right),\left(\phi: \mu_{1} \longrightarrow \mu_{2}\right) \in \operatorname{End}(D, r)$,

$$
\bar{r}(\psi \circ \phi)=r(\psi \circ \phi) r=(r \psi r) \circ(r \phi r)=(\bar{r} \psi) \circ(\bar{r} \phi): \nu_{2} \circ \mu_{2} \rightarrow \nu_{1} \circ \mu_{1} \text {. }
$$




\section{Undirected endomorphism graphs}

In this section we construct functors from Gph and Ugph to Rdgph. In the first case the subcategory Pdgph of pair-free digraphs in Rdgph will be significant. Recall that a subcategory $\mathrm{B}$ of $\mathrm{C}$ is reflective in $\mathrm{C}$ when the inclusion functor $\mathcal{K}: \mathrm{B} \rightarrow \mathrm{C}$ has a left adjoint $\mathcal{F}: \mathrm{C} \rightarrow \mathrm{B}$. There is then a bijection of hom-sets $\mathrm{B}\left(\mathcal{F}_{c}, b\right) \cong \mathrm{C}(c, \mathcal{K} b)$, natural in $c \in \mathrm{C}$ and $b \in \mathrm{B}$ (see [17, IV.3]).

Proposition 7.1 The category Pdgph of pair-free, reversible digraphs is a reflective subcategory of Rdgph.

Proof: Let $\mathcal{F}:$ Rdgph $\rightarrow$ Pdgph be the functor which, when applied to a reversible digraph $D$, has as image a digraph with the same vertices and $\operatorname{arcs}$ as $D$ but with loops of the form $(\{\ell, r \ell\}: u \longrightarrow u)$ for each loop pair $(\ell, r \ell)$ at $u \in V(D)$. This functor $\mathcal{F}$ is the required left-adjoint to the inclusion functor $\mathcal{K}:$ Pdgph $\rightarrow$ Rdgph.

The product of pair-free, reversible digraphs is pair-free. Further, $\left(\Omega, r_{\Omega}\right)$ is pair-free, and so is a subobject classifier for Pdgph. However Pdgph is not closed under internal homs, as the following example shows.

Example 7.2 Let $\left(K_{2}^{2}, r\right) \in$ Pdgph have two vertices, two loops, and four arcs, as shown in the diagram:

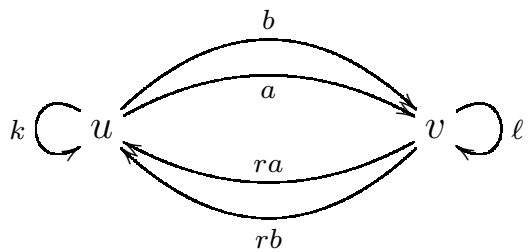

Forgetting the reversal, the automorphism group of $K_{2}^{2}$ is dihedral of order 8 with generating set $\{(a, b),(r a, r b),(u, v)(k, \ell)(a, r a)(b, r b)\}$, and $\operatorname{AUT}\left(K_{2}^{2}\right) \cong K_{8}^{16}$. However, the reversal preserving automorphisms form a subgroup of index 2 , and $\operatorname{AUT}\left(K_{2}^{2}, r\right) \cong K_{4}^{16}$.

Now consider loops in $\operatorname{AUT}\left(K_{2}^{2}, r\right)$ at the identity automorphism. There is a loop $\psi$ with reverse $\theta$ where

\begin{tabular}{|c|cccccccc|}
\hline$c$ & $u$ & $v$ & $k$ & $\ell$ & $a$ & $b$ & $r a$ & $r b$ \\
\hline$\psi(c)$ & $u$ & $v$ & $k$ & $\ell$ & $a$ & $b$ & $r b$ & $r a$ \\
$\theta(c)$ & $u$ & $v$ & $k$ & $\ell$ & $b$ & $a$ & $r a$ & $r b$ \\
\hline
\end{tabular}

So neither $\operatorname{AUT}\left(K_{2}^{2}, r\right)$ nor $\operatorname{END}\left(K_{2}^{2}, r\right)$ are pair-free.

We now define a pair of adjoint functors $\mathcal{R}: \operatorname{Ugph} \rightarrow \operatorname{Rdgph}$ and $\mathcal{U}: \operatorname{Rdgph} \rightarrow$ Ugph, which will enable us to construct a candidate for the endomorphism graph of an undirected graph. 
Definition 7.3 The directing functor $\mathcal{R}:$ Ugph $\rightarrow$ Rdgph constructs a reversible digraph $(D, r)$ from a graph $G$, where

- the vertices of $(D, r)$ are the same as those of $G$;

- there is a directed loop $(\ell: u \longrightarrow u)$ for each loop $(\ell:\{u\}) \in L(G)$;

- there are loops $\left(b^{+}: u \longrightarrow u\right),\left(b^{-}: u \longrightarrow u\right)$ for each band $(b:\{u\}) \in B(G)$;

- there are arcs $((e, u, v): u \longrightarrow v)$ and $((e, v, u): v \longrightarrow u)$ for each edge $(e:\{u, v\}) \in$ $E(G)$.

The reversal $r$ fixes the vertices and the first set of loops; is given on loops coming from bands by $r\left(b^{ \pm}\right)=b^{\mp}$; and on arcs by $r(e, u, v)=(e, v, u)$. If $\mu: G \rightarrow G^{\prime}$ is a morphism of graphs, then the definition of $\mathcal{R}(\mu): \mathcal{R}(G) \rightarrow \mathcal{R}\left(G^{\prime}\right)$ is clear. For example,

$$
\mathcal{R}(\mu)\left(\left(b^{+}: u \longrightarrow u\right)\right)=\left((\mu b)^{+}: \mu u \longrightarrow \mu u\right) .
$$

Definition 7.4 The undirecting functor $\mathcal{U}:$ Rdgph $\rightarrow$ Ugph constructs a graph $G$ from a digraph with reversal $(D, r)$, where

- the vertices of $G$ are the same as those of $(D, r)$;

- there is a loop $(\ell:\{v\})$ for each self-reverse loop $(\ell: v \longrightarrow v)$;

- there is a band $(\{\ell, r \ell\}:\{u\})$ for each reverse pair of loops $\{\ell, r \ell\}$ at $u$;

- there is a single edge $(\{(a, u, v),(r a, v, u)\}:\{u, v\})$ for each pair of arcs $(a: u \longrightarrow v)$ and $(r a: v \longrightarrow u)$.

The definition of $\mathcal{U}(\mu)$ is again clear.

Proposition 7.5 The functors

$$
\mathcal{R}: \text { Ugph } \rightarrow \text { Rdgph, } \quad \mathcal{U}: \text { Rdgph } \rightarrow \text { Ugph }
$$

yield an equivalence of categories.

Proof: If $G$ is a graph, then an isomorphism $G \rightarrow \mathcal{U} \mathcal{R}(G)$ is given by the identity map on vertices and loops, and is given on bands and edges by

$$
\begin{aligned}
(b:\{u\}) & \mapsto\left\{\left(b^{+}: u \rightarrow u\right),\left(b^{-}: u \rightarrow u\right)\right\} \mapsto\left(\left\{b^{+}, b^{-}\right\}:\{u\}\right) \\
(e:\{u, v\}) & \mapsto(\{((e, u, v), u, v),((e, v, u), v, u)\}:\{u, v\}) .
\end{aligned}
$$

Similarly, it is straightforward to verify that the map $(D, r) \rightarrow \mathcal{R} \mathcal{U}(D, r)$ is an isomorphism. 
We are now able to construct a morphism graph UGPH $\left(G, G^{\prime}\right)$. First construct the involutary digraphs $(D, r)=\mathcal{R}(G),\left(D^{\prime}, r^{\prime}\right)=\mathcal{R}\left(G^{\prime}\right)$; then form the morphism digraph $\operatorname{RDGPH}\left((D, r),\left(D^{\prime}, r^{\prime}\right)\right)$; finally, apply $\mathcal{U}$ to obtain the required graph. We have defined a binary operation

$$
\operatorname{UGPH}\left(G, G^{\prime}\right)=\mathcal{U}\left(\operatorname{RDGPH}\left(\mathcal{R}(G), \mathcal{R}\left(G^{\prime}\right)\right)\right) .
$$

The resulting graph has $\operatorname{Ugph}\left(G, G^{\prime}\right)$ as vertex set. When $G, G^{\prime}$ are strict, $\mathcal{R}(G)$ and $\mathcal{R}\left(G^{\prime}\right)$ are both strict and so pair-free, and $\operatorname{UGPH}\left(G, G^{\prime}\right)$ is pair-free.

The endomorphism graph $\operatorname{END}(G)$ is then $\operatorname{UGPH}(G, G)$, and the full subgraph of $\operatorname{END}(G)$ having the automorphisms of $G$ as vertices is the symmetry graph $\operatorname{AUT}(G)$. However, $\operatorname{END}(G)$ and $\operatorname{AUT}(G)$ are not in general an internal monoid or group in Ugph. In particular, the 'product' of two edges

$$
\left\{\left(\psi, \nu_{1}, \nu_{2}\right),\left(r \psi r, \nu_{2}, \nu_{1}\right)\right\} \times\left\{\left(\phi, \mu_{1}, \mu_{2}\right),\left(r \phi r, \mu_{2}, \mu_{1}\right)\right\}
$$

consists of the two edges

$\left\{\left(\psi \phi, \nu_{1} \mu_{1}, \nu_{2} \mu_{2}\right),\left((r \psi r)(r \phi r), \nu_{2} \mu_{2}, \nu_{1} \mu_{1}\right)\right\}, \quad\left\{\left(\psi(r \phi r), \nu_{1} \mu_{2}, \nu_{2} \mu_{1}\right),\left((r \psi r) \phi, \nu_{2} \mu_{1}, \nu_{1} \mu_{2}\right)\right\}$.

Example 7.6 When $G$ is the single edge graph and $\mathcal{R}(G)=(D, r)$,

$$
G: \quad 0 \frac{e}{1} \quad(D, r): \quad 0 \underset{b=(e, 1,0)}{a=(e, 0,1)} 1
$$

then, as in Example 3.1,

$$
\operatorname{AUT}((D, r)): \quad 1_{D} \underset{\phi}{\stackrel{\psi}{\rightleftharpoons} \rho}
$$

where $\rho=(0,1)(a, b), \psi=(0, a)(1, b)$ and $\phi=(0, b)(1, a)$. So the symmetry graph of $G$ is:

$$
\operatorname{AUT}(G): \quad 1_{D} \frac{\left.\left.c=\left\{\left(\psi, 1_{D}, \rho\right\}\right),\left(\phi, 1_{D}, \rho\right\}\right)\right\}}{-} \rho
$$

and the "multiplication table" is

\begin{tabular}{|c|ccc|}
\hline & $1_{D}$ & $\rho$ & $c$ \\
\hline $1_{D}$ & $1_{D}$ & $\rho$ & $c$ \\
$\rho$ & $\rho$ & $1_{D}$ & $c$ \\
$c$ & $c$ & $c$ & $\left\{1_{D}, \rho\right\}$ \\
\hline
\end{tabular}

Finally, we make some definitions for graphs equivalent to those in [23] for digraphs. For $H$ a subgraph of $G$, let $G \backslash H$ denote the full subgraph of $G$ with vertex set $V(G) \backslash V(H)$. Then $H$ is said to be symmetrically embedded in $G$ if every automorphism of $H$ extends 
to an automorphism of $G$ which fixes every edge in $G \backslash H$. An inner subgraph of a graph $G$ is a maximal symmetrically embedded complete subgraph of $G$. The vertex sets of the inner subgraphs form a partition of $V(G)$. An automorphism of a graph $G$ is inner if it restricts to an automorphism of each inner subgraph of $G$.

\section{Proposition 7.7}

(i) If $H$ is an inner subgraph of a graph $G$ then $\mathcal{R}(H)$ is an inner subdigraph of $\mathcal{R}(G)$.

(ii) If $C$ is an inner subdigraph of a reversible digraph $D$ then $\mathcal{U}(C)$ is an inner subgraph of $\mathcal{U}(D)$.

The following result is the equivalent of Theorem 3.3 in [23] for graphs.

Proposition 7.8 The vertices of $\operatorname{AUT}(G)$ which belong to the connected component of the identity automorphism are precisely the inner automorphisms of $G$.

\section{References}

[1] P. Boldi and S. Vigna. Fibrations of graphs. Discrete Math. 243 (1-3) (2002) 21-66.

[2] F. Borceux. Handbook of categorical algebra, volume 50-52 of Encyclopedia of Mathematics and its Applications. Cambridge University Press, Cambridge (1994).

[3] R. Brown. Higher order symmetry of graphs. Irish Math. Soc. Bull. 32 (1994) 46-59.

[4] R. Brown. Topology and Groupoids. Booksurge PLC (2006).

[5] R. Brown, P. J. Higgins and R. Sivera. Nonabelian algebraic topology (2008). A draft of Part 1 is available from: http://www.bangor.ac.uk/ mas010/nonab-a-t.html.

[6] R. Brown and T. Porter. Category theory: an abstract setting for analogy and comparison. In What is Category Theory?, volume 3 of Advanced Studies in Mathematics and Logic, 257-274. Polimetrica Publisher, Italy (2006).

[7] R. T. Bumby and D. M. Latch. Categorical constructions in graph theory. Internat. J. Math. Math. Sci. 9 (1) (1986) 1-16.

[8] M. El-Zahar and N. Sauer. The chromatic number of the product of two 4-chromatic graphs. Combinatorica 5 (1985) 121-126.

[9] P. J. Freyd. Aspect of topoi. Bull. Austral. Math. Soc. 7 (1972) 1-76.

[10] S. M. Gersten. Intersections of finitely generated subgroups of free groups and resolutions of graphs. Invent. Math. 71 (3) (1983) 567-591. Corrigenda ibid, 467-480.

[11] M. Golubitsky and I. Stewart. Nonlinear dynamics of networks: the groupoid formalism. Bull. Amer. Math. Soc. (N.S.) 43 (3) (2006) 305-364. 
[12] P. Hell. An introduction to the category of graphs. In Topics in graph theory (New York, 1977), volume 328 of Ann. New York Acad. Sci., 120-136. New York Acad. Sci., New York (1979).

[13] P. Hell and J. Nešetřil. Graphs and homomorphisms, volume 28 of Oxford Lecture Series in Mathematics and its Applications. Oxford University Press, Oxford (2004).

[14] P. T. Johnstone. Sketches of an elephant: a topos theory compendium, Vol.1. Oxford Logic Guides 43. The Clarendon Press, Oxford University Press, New York (2002).

[15] F. W. Lawvere. Qualitative distinctions between some toposes of generalized graphs. In Categories in computer science and logic (Boulder, CO, 1987), volume 92 of Contemp. Math., 261-299. Amer. Math. Soc., Providence, RI (1989).

[16] F. W. Lawvere and R. Rosebrugh. Sets for mathematics. Cambridge University Press, Cambridge (2003).

[17] S. Mac Lane. Categories for the working mathematician, volume 5 of Graduate Texts in Mathematics. Springer-Verlag, New York, second edition (1998).

[18] S. Mac Lane and I. Moerdijk. Sheaves in geometry and logic. Universitext. SpringerVerlag, New York (1994). A first introduction to topos theory, Corrected reprint of the 1992 edition.

[19] P. Ribenboim. Algebraic structures on graphs. Algebra Universalis 16 (1) (1983) 105-123.

[20] N. Sauer. Hedetniemi's conjecture - a survey. Discrete Math. 229 (2001) 261-292.

[21] J. Shrimpton. Cartesian closed categories of directed graphs. M.Sc. dissertation, University of Wales, Bangor (1988).

[22] J. Shrimpton. Graphs, symmetry and categorical methods. Ph.D. thesis, University of Wales, Bangor (1990).

[23] J. Shrimpton. Some groups related to the symmetry of a directed graph. J. Pure Appl. Algebra 72 (3) (1991) 303-318.

[24] J. R. Stallings. Topology of finite graphs. Invent. Math. 71 (3) (1983) 551-565.

[25] S. Vigna. A guided tour in the topos of graphs. Technical Report 199-97, Università di Milano, Dipartmento di Scienze dell'Informazione (1997). http: //arXiv.org/abs/math.CT/0306394

[26] D. A. Waller. Products of graph projections as a model for multistage communication networks. Electron. Lett. 12 (8) (1976) 206-207.

[27] D. A. Waller. Pullbacks in the category of graphs. In C. Nash-Williams and J. Sheehan, editors, Proceedings of the Fifth British Combinatorial Conference (Univ. Aberdeen, Aberdeen, 1975), 637-642. Congressus Numerantium, No. XV. Utilitas Math., Winnipeg, Man. (1976). 
[28] G. C. Wraith. Lectures on elementary topoi. In Model theory and topoi (Conf., Bangor, 1973), 114-206. Lecture Notes in Math., Vol. 445. Springer, Berlin (1975).

[29] R. T. Živaljević. Groupoids in combinatorics - applications of a theory of local symmetries. In Algebraic and geometric combinatorics, volume 423 of Contemp. Math., 305-324. Amer. Math. Soc., Providence, RI (2006). 